\title{
DETACHMENT LITHOGRAPHY: FABRICATION OF 3D MICROSTRUCTURES AND GUIDED-ASSEMBLY OF NANOMATERIALS
}

\author{
Junghoon Yeom ${ }^{1,2, *}$ and Mark A. Shannon ${ }^{1,2, * *}$ \\ ${ }^{1}$ Department of Mechanical Science and Engineering, University of Illinois, Urbana, Illinois, USA \\ ${ }^{2}$ Cbana Labs Inc., Champaign, Illinois, USA
}

\begin{abstract}
We present a versatile technique to create arrays of micrometer-size patterns of photosensitive polymers like photoresists (PRs) on the surface of soft stamps and to transfer these patterns to planar, curved, and porous substrates. A PR film was patterned through detachment lithography, utilizing the difference in adhesion to induce the fracture in the film. Various $3 \mathrm{D}$ structures were fabricated on glass cylinders as well as flat $\mathrm{Si}$ substrates. The detached patterns were printed on a nanoporous membrane, facilitating the guided assembly of nanomaterials.
\end{abstract}

\section{MAIN TEXT}

Photolithography, despite its dominance in the industries of microelectronics and MEMS for patterning photoresists (PRs), finds a limitation in adapting to curved or 3D substrates due to its reliance on projection optics and the limited depth of focus [1]. Among many recent developments, micro-contact printing ( $\mu \mathrm{CP}$ ) has received considerable attentions to provide a low-cost microfabrication technique as well as produce patterns on complex geometries [2]. The $\mu \mathrm{CP}$ process, however, is normally used to pattern self-assembled monolayers or organic molecules, not PRs that have high molecular weights.

Our approach to the $\mu \mathrm{CP}$ patterning of PRs is to bring a PR film spin-coated on a flat PDMS stamp into intimate contact with a rigid mold with protruding features, and then remove the stamp to pick up the portions of the film that are not in contact (Fig. 1) [3]. This subtractive method for patterning, called detachment lithography (DL), utilizes the mechanical fracture in the film, in which the shear stress for fracture is provided by the difference in adhesion between the mold/film and film/stamp interfaces [4]. The DL process can be harnessed in two ways: i) the detached PR patterns on the stamp can be subsequently printed onto the substrates (right, Fig. 1) and ii) the PR films transferred onto the $\mathrm{Si}$ mold remain photochemically active and can be used to form 3D microstructures (left, Fig. 1).

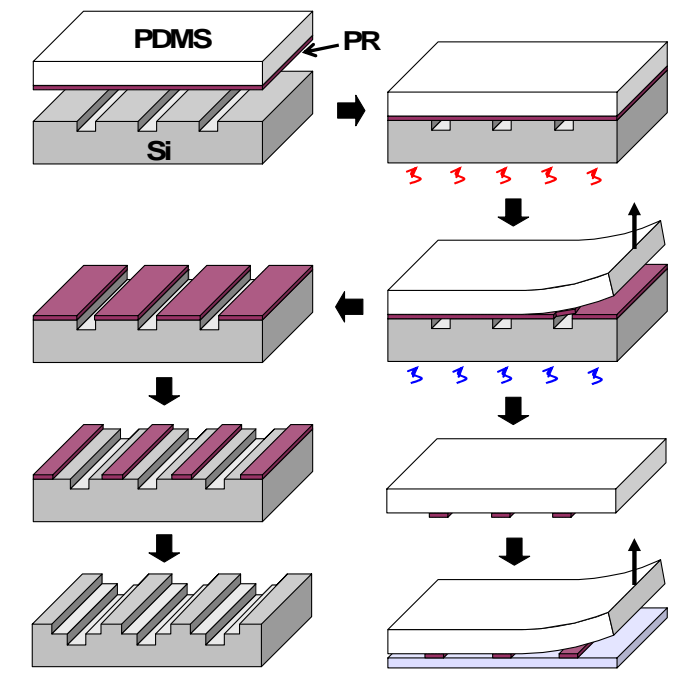

Figure 1: A schematic of the DL process flow.
A thin film of PR $(0.5 \sim 3 \mu \mathrm{m})$ was spin-coated on the flat PDMS stamp, and then the film on the stamp was brought into conformal contact with the pre-patterned Si mold. After annealing, the stamp-PR-mold stack was placed on a cold Al block, and the stamp was removed from the mold at a fast peeling speed $(>10 \mathrm{~cm} / \mathrm{s})$. The regions of the PR film in contact with the protruded parts of the mold were detached, leaving the opposite patterns of the PR on the stamp. The fast removal of the stamp at reduced temperature increased the adhesion between the stamp and film, improving the detachment yield. On the other hand, when the detached film on the stamp was transferred to a receiving substrate, the stamp was removed from the substrate at much slower peeling and an elevated temperature. Thus, the yield of the DL process and ensuing transfer was significantly improved using the kinetically and thermally adjustable adhesion of the elastomeric stamp.

Figure 2(a-b) shows the SEM images of the various detached PR patterns that were printed on the glass slides. The adhesion of the transferred PR films was strong enough to withstand subsequent etching or liftoff process. When a glass cylinder was rolled across the surface of the stamp, the PR patterns were transferred from the stamp to the rod surface (Fig. 2c). The thicker $\mathrm{PR}$ patterns were also transferred to the etched $\mathrm{Si}$ substrate, demonstrating the feasibility of creating free-standing microstructures (Fig. 2d). The minimum feature size attained via the DL process depends on the film thickness, molecular weight, and process parameters (peeling speed and temperature) [5]. For S1805 of $0.56 \mu \mathrm{m}$ in thickness, as small as $5-\mu \mathrm{m}$ lines separated by $5-\mu \mathrm{m}$ could be detached and transferred. The completeness of detachment was investigated with XPS and micro Raman spectroscopy. The element scan from XPS showed no residual PR from the detached region, and Raman signal for PR was not detected from the PDMS stamp after detachment.
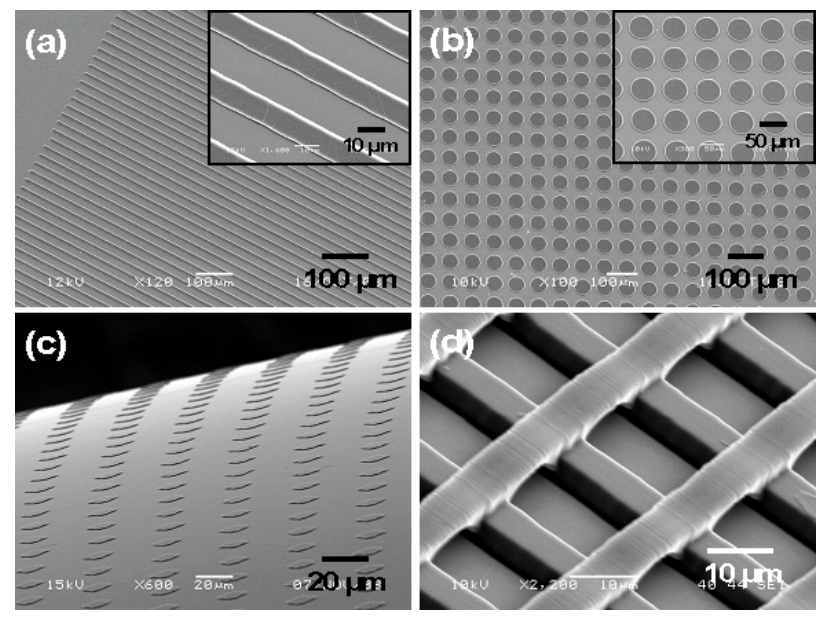

Figure 2: The detached $P R$ patterns are transferred to planar, curved, and protruded substrates. SEM images of 0.85- $\mu m$-thick $P R$ S1805 are shown for an array of (a) $10 \mu \mathrm{m}$ lines separated by $20 \mu \mathrm{m}$, (b) $50 \mu \mathrm{m}$ circles separated by $20 \mu \mathrm{m}$ printed on glass slides, (c) $10 \times 10 \mu \mathrm{m}$ squares transferred on a glass cylinder of $6 \mathrm{~mm}$ in dia., and (d) $7 \mu \mathrm{m}$ lines tenting over the etched patterns. 

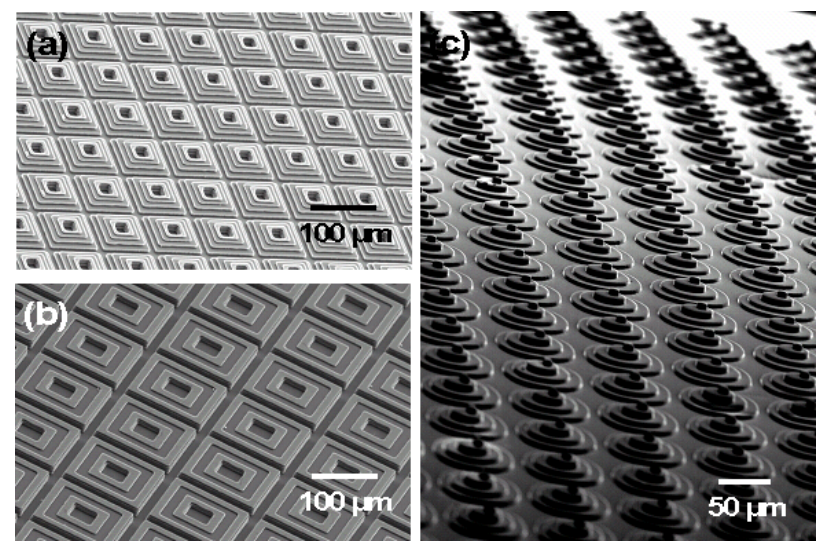

Figure 3: SEM images of an array of (a-b) 4-level $\mathrm{Si}$ microstructures using DRIE etching with the transferred $P R$ as a mask, (c) multilevel PDMS microstructures molded from the Si microstructures and bonded onto a glass cylinder.

The transferred PR films on the Si mold in Fig. 1 remain photochemically active and can be patterned using standard photolithography. PR 1518 was transferred only to the top surface of the pre-etched $\mathrm{Si}$, and the transferred film was uniform across the 5-cm wafer area. After exposure and development, the patterned PR served as an etch mask, and the already-etched Si substrate was further etched using DRIE to form Si multilevel structures (Fig. 3a-b). These 3D structures are difficult to be fabricated because non-uniform coating of PR is generated when spin-coated or sprayed. The smallest features in the 3D structures we fabricated were as small as $2-\mu \mathrm{m}$, and the submicron 3D structures could be generated if the proper masks were available. The 3D structures on the curved surface were also created by the PDMS molded from the multilayer Si structures and bonded on a glass rod (Fig. 3c).

Another challenging substrate to be patterned is a porous membrane. If the PR solution is spin-coated or sprayed, the PR would flow into the pores, making it difficult to be exposed and developed. Moreover, many porous substrates are fragile and delicate, and the lamination with dry photoresist films may damage the porous membrane or substrate. With the DL process, the PR patterns picked up by the stamp can be printed onto the porous membrane without damaging or plugging the pores.

As a proof-of-a-concept, the detached PR patterns were transferred to the anodized aluminum oxide (AAO) membrane (Fig. 4a). The utility of the patterned porous membrane was demonstrated when various nanomaterials in solution were vacuum-filtered through the membrane [6]. The nanomaterials would deposit only on the windows of the patterned membrane, which may represent a facile, robust, and inexpensive assembly method of nanomaterials. The single-walled carbon nanotubes and latex particles of $\sim 1 \mu \mathrm{m}$ in solutions were assembled in the patterned AAO membrane as shown in Fig. 4b and c, respectively.

\section{ACKNOWLEDGEMENT}

The authors acknowledge the financial support from the NANOCEMMS at the University of Illinois, which is funded by National Science Foundation under Grant DMI-0328162. We also thank the travel support from Cbana Labs Inc.

\section{CONTACT}

*J. Yeom; jungyeom@illinois.edu,

***A. Shannon; mshannon@illinois.edu
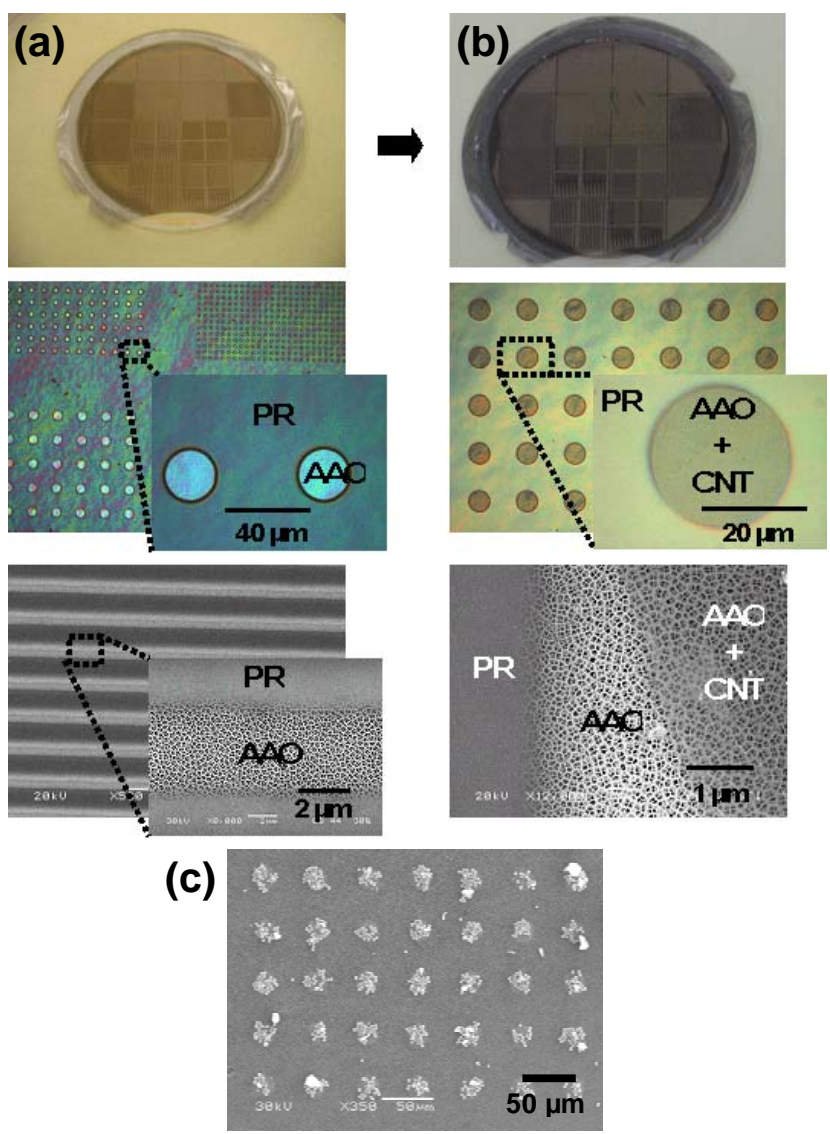

Figure 4: (a) the detached PR patterns transferred to a $220 \mathrm{~nm}$ anodized aluminum oxide (AAO) filter substrate, (b) single-walled carbon nanotubes (SWCNT) suspended in 1\% SDS solution were vacuum-filtrated over the patterned $A A O$ membrane, and (c) a $S E M$ image of an array of $1 \mu \mathrm{m}$ latex beads filtrated over the patterned AAO membrane.

\section{REFERENCES}

[1] R.J. Jackman, J.L. Wilbur, and G.M. Whitesides, "Fabrication of Submicrometer Features on Curved Substrates by Microcontact Printing”, Science, 269, 664-666 (1995)

[2] Y. Xia and G.M. Whitesides, "Soft Lithography", Angewandte Chemie International Edition, 37, 5, 550-575 (1998)

[3] J. Yeom and M.A. Shannon, "Detachment Lithography of Photosensitive Polymers: A Route to Fabricating Threedimensional Structures”, Advanced Functional Materials, 20, 2, 289-295 (2010)

[4] J.-H. Choi, D. Kim, P.J. Yoo, and H.H. Lee, "Simple Detachment Patterning of Organic Layers and its Application to Organic Light-emitting Diodes”, Advanced Materials, 17, 2, 166-171 (2005)

[5] M.A. Meitl, Z.-T. Zhu, V. Kumar, K.J. Lee, X. Feng, Y.Y. Huang, I. Adesida, R.G. Nuzzo, and J.A. Rogers, "Transfer Printing by Kinetic Control of Adhesion to an Elastomeric Stamp”, Nature Materials, 5, 1, 33-38 (2006)

[6] J.H. Oh, H.W. Lee, S. Mannsfeld, R.M. Stoltenberg, E. Jung, Y.W. Jin, J.M. Kim, J.-B. Yoo, and Z. Bao, "SolutionProcessed, High-performance n-Channel Organic Microwire Transistors", Proceedings of the National Academy of Sciences, 106, 15, 6065-6070 (2009) 